Research Article

\title{
System of Nonlinear Set-Valued Variational Inclusions Involving a Finite Family of $H(\cdot, \cdot)$-Accretive Operators in Banach Spaces
}

\author{
Prapairat Junlouchai and Somyot Plubtieng \\ Department of Mathematics, Faculty of Science, Naresuan University, Phitsanulok 65000, Thailand \\ Correspondence should be addressed to Somyot Plubtieng, somyotp@nu.ac.th
}

Received 15 February 2012; Accepted 27 March 2012

Academic Editor: Giuseppe Marino

Copyright (c) 2012 P. Junlouchai and S. Plubtieng. This is an open access article distributed under the Creative Commons Attribution License, which permits unrestricted use, distribution, and reproduction in any medium, provided the original work is properly cited.

We study a new system of nonlinear set-valued variational inclusions involving a finite family of $H(\cdot, \cdot)$-accretive operators in Banach spaces. By using the resolvent operator technique associated with a finite family of $H(\cdot, \cdot)$-accretive operators, we prove the existence of the solution for the system of nonlinear set-valued variational inclusions. Moreover, we introduce a new iterative scheme and prove a strong convergence theorem for finding solutions for this system.

\section{Introduction}

Variational inequality theory has become a very effective and powerful tool for studying a wide range of problems arising in pure and applied sciences which include work on differential equations, control problems, mechanics, general equilibrium problems in transportation and economics. In 1994, Hassouni and Moudafi [1] introduced and studied a class of variational inclusions and developed a perturbed algorithm for finding approximate solutions of the variational inclusions. In 1996, Adly [2] obtained some important extensions and generalizations of the results in [1] for nonlinear variational inclusions. Recently, Ding [3] introduced and studied a class of generalized quasivariational inclusions and Kazmi [4] introduced and studied another class of quasivariational inclusions in the same year. In $[5,6]$, Ansari et al. introduced the system of vector equilibrium problems and they proved the existence of solutions for such problems (see also in [7-9]). In 2004, Verma [10] studied nonlinear variational inclusion problems based on the generalized resolvent operator technique involving $A$-monotone mapping. For existence result and approximating solution of the system of set-valued variational inclusions and the class of nonlinear relaxed cocoercive 
variational inclusions, we refer the reader to Yan et al. [11], Plubtieng and Sriprad [12], Verma [13] and Cho et al. [14].

Very recently, Verma [15] introduced and studied approximation solvability of a general class of nonlinear variational inclusion problems based on $(A, \eta)$-resolvent operator technique in a Hilbert space. On the other hand, Zou and Huang [16] studied the Lipschitz continuity of resolvent operator for the $H(\cdot, \cdot)$-accretive operator in Banach spaces. Moreover, they also applied these new concepts to solve a variational-like inclusion problem. One year later, Zou and Huang [17] introduced and studied a new class of system of variational inclusions involving $H(\cdot, \cdot)$-accretive operator in Banach spaces. By using the resolvent operator technique associated with $H(\cdot, \cdot)$-accretive operator, they proved the existence of the solution for the system of inclusions. Moreover, they also develop a step-controlled iterative algorithm to approach the unique solution.

In this paper, we introduce a new system of nonlinear set-valued variational inclusions involving a finite family of $H(\cdot, \cdot)$-accretive operators in Banach spaces. By using the resolvent operators technique associated with a finite family of $H(\cdot, \cdot)$-accretive operator, we prove the existence of the solution for the system of nonlinear set-valued variational inclusions. Moreover, we introduce a new iterative scheme and prove a strong convergence theorem for finding solutions of this system.

\section{Preliminaries}

Let $X$ be a real Banach space with dual space $X^{*},\langle\cdot, \cdot\rangle$ the dual pair between $X$ and $X^{*}$ and $2^{X}$ and $C(X)$ denote the family of all the nonempty subsets of $X$ and the family of all closed subsets of $X$, respectively. The generalized duality mapping $J_{q}: X \rightarrow 2^{X^{*}}$ is defined by

$$
J_{q}(X)=\left\{f^{*} \in X^{*}:\left\langle x, f^{*}\right\rangle=\|x\|^{q},\left\|f^{*}\right\|=\|x\|^{q-1}\right\}, \quad \forall x \in X,
$$

where $q>1$ is a constant. It is known that, in general, $J_{q}(x)=\|x\|^{q-1} J_{2}(x)$ for all $x \neq 0$ and $J_{q}$ is single-valued if $X^{*}$ is strictly convex. In the sequel, we always assume that $X$ is a real Banach space such that $J_{q}$ is single-valued.

The modulus of smoothness of $X$ is the function $\rho_{X}:[0, \infty) \rightarrow[0, \infty)$ defined by

$$
\rho_{X}(t)=\sup \left\{\frac{\|x+y\|+\|x-y\|}{2}-1:\|x\| \leq 1,\|y\| \leq t\right\} .
$$

A Banach space $X$ is called uniformly smooth if

$$
\lim _{t \rightarrow 0} \frac{\rho_{X}(t)}{t}=0
$$

$X$ is called $q$-uniformly smooth if there exists a constant $c>0$ such that

$$
\rho_{X}(t) \leq c t^{q}, \quad q>1 \text {. }
$$


Note that $J_{q}$ is single valued if $X$ is uniformly smooth. In the study of characteristic inequalities in $q$-uniformly smooth Banach spaces, $\mathrm{Xu}$ [18] proved the following result.

Definition 2.1. Let $H, \eta: X \times X \rightarrow X$ be two single-valued mappings and $A, B: X \rightarrow X$ two single-valued mappings.

(i) $A$ is said to be accretive if

$$
\left\langle A x-A y, J_{q}(x-y)\right\rangle \geq 0, \quad \forall x, y \in X,
$$

(ii) $A$ is said to be strictly accretive if $A$ is accretive and

$$
\left\langle A x-A y, J_{q}(x-y)\right\rangle=0, \quad \forall x, y \in X,
$$

if and only if $x=y$;

(iii) $H(A, \cdot)$ is said to be $\alpha$-strongly accretive with respect to $A$ if there exists a constant $\alpha>0$ such that

$$
\left\langle H(A x, u)-H(A y, u), J_{q}(x-y)\right\rangle \geq \alpha\|x-y\|^{q}, \quad \forall x, y, u \in X
$$

(iv) $H(\cdot, B)$ is said to be $\beta$-relaxed accretive with respect to $B$ if there exists a constant $\beta>0$ such that

$$
\left\langle H(u, B x)-H(u, B y), J_{q}(x-y)\right\rangle \geq-\beta\|x-y\|^{q}, \quad \forall x, y, u \in X
$$

(v) $H(\cdot, \cdot)$ is said to be $\gamma$-Lipschitz continuous with respect to $A$ if there exists a constant $r>0$ such that

$$
\|H(A x, u)-H(A y, u)\| \leq r\|x-y\|^{q}, \quad \forall x, y, u \in X
$$

(vi) $A$ is said to be $\theta$-Lipschitz continuous if there exists a constant $\theta>0$ such that

$$
\|A x-A y\| \leq \theta\|x-y\|^{q}, \quad \forall x, y \in X
$$


(vii) $\eta(\cdot, \cdot)$ is said to be strongly accretive with respect to $H(A, B)$ if there exists a constant $\rho>0$ such that

$$
\left\langle\eta(x, u)-\eta(y, u), J_{q}(H(A x, B x)-H(A y, B y))\right\rangle \geq \rho\|x-y\|^{q}, \quad \forall x, y, u \in X .
$$

Definition 2.2. Let $\eta: X \times X \rightarrow X$ be single-valued mapping. Let $M: X \rightarrow 2^{X}$ be a set-valued mapping.

(i) $\eta$ is said to be $\tau$-Lipschitz continuous if there exists a constant $\tau>0$ such that

$$
\|\eta(x, y)\| \leq \tau\|x-y\|, \quad \forall x, y \in X
$$

(ii) $M$ is said to be accretive if

$$
\left\langle u-v, J_{q}(x-y)\right\rangle \geq 0, \quad \forall x, y \in X, u \in M(x), v \in M(y)
$$

(iii) $M$ is said to be $\eta$-accretive if

$$
\left\langle u-v, J_{q}(\eta(x, y))\right\rangle \geq 0, \quad \forall x, y \in X, u \in M(x), v \in M(y)
$$

(iv) $M$ is said to be strictly $\eta$-accretive if $M$ is $\eta$-accretive and equality holds if and only if $x=y$;

(v) $M$ is said to be $\gamma$-strongly $\eta$-accretive if there exists a positive constant $\gamma>0$ such that

$$
\left\langle u-v, J_{q}(\eta(x, y))\right\rangle \geq \gamma\|x-y\|^{q}, \quad \forall x, y \in X, u \in M(x), v \in M(y)
$$

(vi) $M$ is said to be $\alpha$-relaxed $\eta$-accretive if there exists a positive constant $\alpha>0$ such that

$$
\left\langle u-v, J_{q}(\eta(x, y))\right\rangle \geq-\alpha\|x-y\|^{q}, \quad \forall x, y \in X, u \in M(x), v \in M(y)
$$


Definition 2.3. Let $A, B: X \rightarrow X, H: X \times X \rightarrow X$ be three single-valued mappings. Let $M: X \rightarrow 2^{X}$ be a set-valued mapping. $M$ is said to be $H(\cdot, \cdot)$-accretive with respect to $A$ and $B$ (or simply $H(\cdot, \cdot)$-accretive in the sequel), if $M$ is accretive and $(H(A, B)+\lambda M)(X)=X$ for every $\lambda>0$.

Lemma 2.4. Let $X$ be a real uniformly smooth Banach space. Then $X$ is q-uniformly smooth if and only if there exists a constant $c_{q}>0$ such that for all $x, y \in X$

$$
\|x+y\|^{q} \leq\|x\|^{q}+q\left\langle y, J_{q}(x)\right\rangle+c_{q}\|y\|^{q} .
$$

Lemma 2.5 (see[16]). Let $H(A, B)$ be $\alpha$-strongly accretive with respect to $A$, $\beta$-relaxed accretive with respect to $B$, and $\alpha>\beta$. Let $M$ be an $H(\cdot, \cdot)$-accretive operator with respect to $A$ and $B$. Then, the operator $H((A, B)+\lambda M)^{-1}$ is single valued. Based on Lemma 2.4, one can define the resolvent operator $R_{M, i}^{H(, \cdot)}$ as follows.

Definition 2.6. Let $H, A, B, M$ be defined as in Definition 2.3. Let $H(A, B)$ be $\alpha$-strongly accretive with respect to $A, \beta$-relaxed accretive with respect to $B$, and $\alpha>\beta$. Let $M$ be an $H(\cdot, \cdot)$-accretive operator with respect to $A$ and $B$. The resolvent operator $R_{M, \lambda}^{H(\cdot, \cdot)}: X \rightarrow X$ is defined by

$$
R_{M, \lambda}^{H(\cdot, \cdot)}(z)=(H(A, B)+\lambda M)^{-1}(z), \quad \forall z \in X
$$

where $\ell>0$ is a constant.

Lemma 2.7 (see [16]). Let $H, A, B, M$ be defined as in Definition 2.3. Let $H(A, B)$ be $\alpha$-strongly accretive with respect to $A, \beta$-relaxed accretive with respect to $B$, and $\alpha>\beta$. Suppose that $M: X \rightarrow$ $2^{\mathrm{X}}$ is an $H(\cdot, \cdot)$-accretive operator. Then resolvent operator $R_{M, \lambda}^{H(\cdot, \cdot)}$ defined by $(2.18)$ is $1 /(\alpha-\beta)$ Lipschitz continuous. That is,

$$
\left\|R_{M, \lambda}^{H(\cdot,)}(x)-R_{M, \lambda}^{H(\cdot, \cdot)}(y)\right\| \leq \frac{1}{\alpha-\beta}\|x-y\|, \quad \forall x, y \in X
$$

We define a Hausdorff pseudometric $D: 2^{X} \times 2^{X} \rightarrow[0,+\infty]$ by

$$
D(U, V)=\max \left\{\sup _{u \in U} \inf _{v \in V}\|u-v\|, \sup _{u \in V} \inf _{v \in U}\|u-v\|\right\}
$$

for any given $U, V \in 2^{X}$. Note that if the domain of $D$ is restricted to closed bounded subsets, then $D$ is the Hausdorff metric.

Lemma 2.8 (see [19]). Let $\left\{c_{n}\right\}$ and $\left\{k_{n}\right\}$ be two real sequences of nonnegative numbers that satisfy the following conditions:

(i) $0<k_{n}<1$ for $n=0,1,2, \ldots$, and $\lim \sup _{n} k_{n}<1$;

(ii) $c_{n+1} \leq k_{n} c_{n}$ for $n=0,1,2, \ldots$

Then, $c_{n}$ converges to 0 as $n \rightarrow \infty$. 


\section{Main Result}

Let $X$ be $q$-uniformly smooth real Banach space and $C(X)$ a nonempty closed convex set. Let $S_{i}, H_{i}: X \times X \rightarrow X, A_{i}, B_{i}: X \rightarrow X$ be single-valued operators, for all $i=1,2, \ldots, N$. For any fix $i \in\{1,2, \ldots, N\}$, we let $M_{i}: X \rightarrow 2^{X}, H_{i}\left(A_{i}, B_{i}\right)$-accretive set-valued operator and $U_{i}: X \rightarrow 2^{X}$ a set-valued mapping which nonempty values. The system of nonlinear setvalued variational inclusions is to find $a_{1}, \ldots, a_{N} \in X, u_{1} \in U_{1}\left(a_{N}\right), \ldots, u_{N} \in U_{N}\left(a_{1}\right)$ such that

$$
0 \in S_{i}\left(a_{i}, u_{i}\right)+M_{i}\left(a_{i}\right), \quad \forall i=1,2, \ldots, N
$$

If $N=2$, then system of nonlinear set-valued variational inclusions (3.1) becomes following system of variational inclusions: finding $a_{1}, a_{2} \in X, u_{1} \in U_{1}\left(a_{2}\right)$ and $u_{2} \in U_{2}\left(a_{1}\right)$ such that

$$
\begin{aligned}
& 0 \in S_{1}\left(a_{1}, u_{1}\right)+M_{1}\left(a_{1}\right), \\
& 0 \in S_{2}\left(a_{2}, u_{2}\right)+M_{2}\left(a_{2}\right) .
\end{aligned}
$$

If $N=1$, then system of nonlinear set-valued variational inclusions (3.1) becomes the following class of nonlinear set-valued variational inclusions see [15]: finding $a \in X$, $u \in U(a)$ such that

$$
0 \in S(a, u)+M(a)
$$

For solving the system of nonlinear set-valued variational inclusions involving a finite family of $H(\cdot, \cdot)$-accretive operators in Banach spaces, let us give the following assumptions.

For any $i \in\{1,2, \ldots, N\}$, we suppose that

(A1) $H\left(A_{i}, B_{i}\right)$ is $\alpha_{i}$-strongly accretive with respect to $A_{i}, \beta_{i}$-relaxed accretive with respect to $B_{i}$ and $\alpha_{i}>\beta_{i}$,

(A2) $M_{i}: X \rightarrow 2^{X}$ is an $H_{i}(\cdot, \cdot)$-accretive single-valued mapping,

(A3) $U_{i}: X \rightarrow C(X)$ is a contraction set-valued mapping with $0 \leq L_{i}<1$ and nonempty values,

(A4) $H_{i}\left(A_{i}, B_{i}\right)$ is $r_{i}$-Lipschitz continuous with respect to $A_{i}$ and $t_{i}$-Lipschitz continuous with respect to $B_{i}$

(A5) $S_{i}: X \times X \rightarrow X$ is $l_{i}$-Lipschitz continuous with respect to its first argument and $m_{i}$-Lipschitz continuous with respect to its second argument,

(A6) $S_{i}(\cdot, u)$ is $s_{i}$-strongly accretive with respect to $H_{i}\left(A_{i}, B_{i}\right)$.

Theorem 3.1. For given $a_{1}, \ldots, a_{N} \in X, u_{1} \in U_{1}\left(a_{N}\right), \ldots, u_{N} \in U_{N}\left(a_{1}\right)$, it is a solution of problem (3.1) if and only if

$$
a_{i}=R_{M_{i}, \lambda_{i}}^{H_{i}(\cdot)}\left[H_{i}\left(A_{i}\left(a_{i}\right), B_{i}\left(a_{i}\right)\right)-\lambda_{i} S_{i}\left(a_{i}, u_{i}\right)\right]
$$

where $\lambda_{i}>0$ are constants. 
Proof. We note from the Definition 2.6 that $a_{1}, \ldots, a_{N} \in X, u_{1} \in U_{1}\left(a_{N}\right), \ldots, u_{N} \in U_{N}\left(a_{1}\right)$ is a solution of (3.1) if and only if, for each $i \in\{1,2, \ldots, N\}$, we have

$$
\begin{aligned}
a_{i} & =R_{M_{i}, \lambda_{i}}^{H_{i}(\cdot, \cdot)}\left[H_{i}\left(A_{i}\left(a_{i}\right), B_{i}\left(a_{i}\right)\right)-\lambda_{i} S_{i}\left(a_{i}, u_{i}\right)\right] \\
& \Longleftrightarrow a_{i}=\left[H_{i}\left(A_{i}, B_{i}\right)+\lambda_{i} M_{i}\right]^{-1}\left[H_{i}\left(A_{i}\left(a_{i}\right), B_{i}\left(a_{i}\right)\right)-\lambda_{i} S_{i}\left(a_{i}, u_{i}\right)\right] \\
& \Longleftrightarrow\left[H_{i}\left(A_{i}\left(a_{i}\right), B_{i}\left(a_{i}\right)\right)-\lambda_{i} S_{i}\left(a_{i}, u_{i}\right)\right] \in\left[H_{i}\left(A_{i}, B_{i}\right)+\lambda_{i} M_{i}\right]\left(a_{i}\right) \\
& \Longleftrightarrow-\lambda_{i} S_{i}\left(a_{i}, u_{i}\right) \in \lambda_{i} M_{i}\left(a_{i}\right) \\
& \Longleftrightarrow 0 \in S_{i}\left(a_{i}, u_{i}\right)+M_{i}\left(a_{i}\right) .
\end{aligned}
$$

Algorithm 3.2. For given $a_{0}^{1}, \ldots, a_{0}^{N} \in X, u_{0}^{1} \in U_{1}\left(a_{0}^{N}\right), \ldots, u_{0}^{N} \in U_{N}\left(a_{0}^{1}\right)$, we let

$$
a_{1}^{i}=\sigma_{0} a_{0}^{i}+\left(1-\sigma_{0}\right) R_{M_{i}, \lambda_{i}}^{H_{i}(\cdot)}\left[H_{i}\left(A_{i}\left(a_{0}^{i}\right), B_{i}\left(a_{0}^{i}\right)\right)-\lambda_{i} S_{i}\left(a_{0}^{i}, u_{0}^{i}\right)\right]
$$

for all $i=1,2, \ldots, N$, where $0<\sigma_{0} \leq 1$. By Nadler theorem [20], there exists $u_{1}^{1} \in U_{1}$ $\left(a_{1}^{N}\right), \ldots, u_{1}^{N} \in U_{N}\left(a_{1}^{1}\right)$ such that

$$
\left\|u_{1}^{i}-u_{0}^{i}\right\| \leq(1+1) D\left(U_{i}\left(a_{1}^{N-(i-1)}\right), U_{i}\left(a_{0}^{N-(i-1)}\right)\right), \quad \forall i=1,2, \ldots, N
$$

where $D(\cdot, \cdot)$ is the Hausdorff pseudo metric on $2^{X}$. Continuing the above process inductively, we can obtain the sequences $\left\{a_{n}^{i}\right\}$ and $\left\{u_{n}^{i}\right\}$ such that

$$
a_{n+1}^{i}=\sigma_{n} a_{n}^{i}+\left(1-\sigma_{n}\right) R_{M_{i}, \lambda_{i}}^{H_{i}(\cdot)}\left[H_{i}\left(A_{i}\left(a_{n}^{\mathrm{i}}\right), B_{i}\left(a_{n}^{i}\right)\right)-\lambda_{i} S_{i}\left(a_{n}^{i}, u_{n}^{i}\right)\right]
$$

for all $n=1,2,3, \ldots, i=1,2, \ldots, N$, where $0<\sigma_{n} \leq 1$ with $\lim _{\sup _{n \rightarrow \infty}} \sigma_{n}<1$. Therefore, by Nadler theorem [20], there exists $u_{n+1}^{1} \in U_{1}\left(a_{n+1}^{N}\right), \ldots, u_{n+1}^{N} \in U_{N}\left(a_{n+1}^{1}\right)$ such that

$$
\begin{aligned}
& \left\|u_{n+1}^{i}-u_{n}^{i}\right\| \leq\left(1+(1+n)^{-1}\right) D\left(U_{i}\left(a_{n+1}^{N-(i-1)}\right),\right. \\
& \left.U_{i}\left(a_{n}^{N-(i-1)}\right)\right), \quad \forall n=1,2,3, \ldots, i=1,2, \ldots, N .
\end{aligned}
$$

The idea of the proof of the next theorem is contained in the paper of Verma [15] and Zou and Huang [17]. 
Theorem 3.3. Let $X$ be q-uniformly smooth real Banach space. Let $A_{i}, B_{i}: X \rightarrow X$ be single-valued operators, $H_{i}: X \times X \rightarrow X$ a single-valued operator satisfy (A1) and $M_{i}, U_{i}, H_{i}\left(A_{i}, B_{i}\right), S_{i}, S_{i}(\cdot, u)$ satisfy conditions $(A 2)-(A 6)$, respectively. If there exists a constant $c_{q, i}$ such that

$$
\frac{\sqrt[q]{\left(r_{i}+t_{i}\right)^{q}-q \lambda_{i} s_{i}+c_{q, i} \lambda_{i}^{q} l_{i}^{q}}}{\alpha_{i}-\beta_{i}}+\frac{\lambda_{i} m_{i}}{\alpha_{i}-\beta_{i}}<1
$$

for all $i=1,2, \ldots, N$, then problem (3.1) has a solution $a_{1}, \ldots, a_{N}, u_{1} \in U_{1}\left(a_{N}\right), \ldots, u_{N} \in U_{N}\left(a_{1}\right)$.

Proof. For any $i \in\{1,2, \ldots, N\}$ and $\lambda_{i}>0$, we define $F_{i}: X \times X \rightarrow X$ by

$$
F_{i}(u, v)=R_{M_{i}, \lambda_{i}}^{H_{i}(\cdot, \cdot)}\left[H_{i}\left(A_{i}(u), B_{i}(u)\right)-\lambda_{i} S_{i}(u, v)\right],
$$

for all $u, v \in X$. Let $J_{i}(x, y)=H_{i}\left(A_{i}(x), B_{i}(y)\right)$. For any $\left(u_{1}, v_{1}\right),\left(u_{2}, v_{2}\right) \in X \times X$, we note by (3.11) and Lemma 2.7 that

$$
\begin{aligned}
\left\|F_{i}\left(u_{1}, v_{1}\right)-F_{i}\left(u_{2}, v_{2}\right)\right\|= & \| R_{M_{i}, \lambda_{i}}^{H_{i}(\cdot, \cdot)}\left[H_{i}\left(A_{i}\left(u_{1}\right), B_{i}\left(u_{1}\right)\right)-\lambda_{i} S_{i}\left(u_{1}, v_{1}\right)\right] \\
& -R_{M_{i}, \lambda_{i}}^{H_{i}(\cdot, \cdot)}\left[H_{i}\left(A_{i}\left(u_{2}\right), B_{i}\left(u_{2}\right)\right)-\lambda_{i} S_{i}\left(u_{2}, v_{2}\right)\right] \| \\
= & \left\|R_{M_{i}, \lambda_{i}}^{H_{i}(\cdot)}\left[J_{i}\left(u_{1}, u_{1}\right)-\lambda_{i} S_{i}\left(u_{1}, v_{1}\right)\right]-R_{M_{i}, \lambda_{i}}^{H_{i}(\cdot,)}\left[J_{i}\left(u_{2}, u_{2}\right)-\lambda_{i} S_{i}\left(u_{2}, v_{2}\right)\right]\right\| \\
\leq & \frac{1}{\alpha_{i}-\beta_{i}}\left\|\left[J_{i}\left(u_{1}, u_{1}\right)-\lambda_{i} S_{i}\left(u_{1}, v_{1}\right)\right]-\left[J_{i}\left(u_{2}, u_{2}\right)-\lambda_{i} S_{i}\left(u_{2}, v_{2}\right)\right]\right\| \\
= & \frac{1}{\alpha_{i}-\beta_{i}}\left\|\left[J_{i}\left(u_{1}, u_{1}\right)-J_{i}\left(u_{2}, u_{2}\right)\right]-\lambda_{i}\left[S_{i}\left(u_{1}, v_{1}\right)-S_{i}\left(u_{2}, v_{2}\right)\right]\right\| \\
\leq & \frac{1}{\alpha_{i}-\beta_{i}}\left\|\left[J_{i}\left(u_{1}, u_{1}\right)-J_{i}\left(u_{2}, u_{2}\right)\right]-\lambda_{i}\left[S_{i}\left(u_{1}, v_{1}\right)-S_{i}\left(u_{2}, v_{1}\right)\right]\right\| \\
& +\frac{\lambda_{i}}{\alpha_{i}-\beta_{i}}\left\|\left[S_{i}\left(u_{2}, v_{1}\right)-S_{i}\left(u_{2}, v_{2}\right)\right]\right\| .
\end{aligned}
$$

By Lemma 2.4, we have

$$
\begin{aligned}
&\left\|J_{i}\left(u_{1}, u_{1}\right)-J_{i}\left(u_{2}, u_{2}\right)-\lambda_{i}\left[S_{i}\left(u_{1}, v_{1}\right)-S_{i}\left(u_{2}, v_{1}\right)\right]\right\|^{q} \\
& \leq\left\|J_{i}\left(u_{1}, u_{1}\right)-J_{i}\left(u_{2}, u_{2}\right)\right\|^{q} \\
&-q \lambda_{i}\left\langle S_{i}\left(u_{1}, v_{1}\right)-S_{i}\left(u_{2}, v_{1}\right), J_{q}\left(J_{i}\left(u_{1}, u_{1}\right)-J_{i}\left(u_{2}, u_{2}\right)\right)\right\rangle \\
&+c_{q, i} \lambda_{i}^{q}\left\|S_{i}\left(u_{1}, v_{1}\right)-S_{i}\left(u_{2}, v_{1}\right)\right\|^{q} .
\end{aligned}
$$


Moreover, by (A4), we obtain

$$
\begin{aligned}
\left\|J_{i}\left(u_{1}, u_{1}\right)-J_{i}\left(u_{2}, u_{2}\right)\right\| & \leq\left\|J_{i}\left(u_{1}, u_{1}\right)-J_{i}\left(u_{2}, u_{1}\right)\right\|+\left\|J_{i}\left(u_{2}, u_{1}\right)-J_{i}\left(u_{2}, u_{2}\right)\right\| \\
& \leq r_{i}\left\|u_{1}-u_{2}\right\|+t_{i}\left\|u_{1}-u_{2}\right\| \\
& \leq\left(r_{i}+t_{i}\right)\left\|u_{1}-u_{2}\right\| .
\end{aligned}
$$

From (A6), we have

$$
-q \lambda_{i}\left\langle S_{i}\left(u_{1}, v_{1}\right)-S_{i}\left(u_{2}, v_{1}\right), J_{q}\left(J_{i}\left(u_{1}, u_{1}\right)-J_{i}\left(u_{2}, u_{2}\right)\right)\right\rangle \leq-q \lambda_{i} s_{i}\left\|u_{1}-u_{2}\right\|^{q} .
$$

Moreover, from (A5), we obtain

$$
\begin{aligned}
& \left\|S_{i}\left(u_{1}, v_{1}\right)-S_{i}\left(u_{2}, v_{1}\right)\right\| \leq l_{i}\left\|u_{1}-u_{2}\right\|, \\
& \left\|S_{i}\left(u_{2}, v_{1}\right)-S_{i}\left(u_{2}, v_{2}\right)\right\| \leq m_{i}\left\|v_{1}-v_{2}\right\| .
\end{aligned}
$$

From (3.13)-(3.16), we have

$$
\left\|J_{i}\left(u_{1}, u_{1}\right)-J_{i}\left(u_{2}, u_{2}\right)-\lambda_{i}\left[S_{i}\left(u_{1}, v_{1}\right)-S_{i}\left(u_{2}, v_{1}\right)\right]\right\|^{q} \leq \sqrt[q]{\left(r_{i}+t_{i}\right)^{q}-q \lambda_{i} S_{i}+c_{q, i} i l_{i}^{q} l_{i}^{q}}\left\|u_{1}-u_{2}\right\| .
$$

It follows from (3.12), (3.17), and (3.18) that

$$
\left\|F_{i}\left(u_{1}, v_{1}\right)-F_{i}\left(u_{2}, v_{2}\right)\right\| \leq \frac{\sqrt[q]{\left(r_{i}+t_{i}\right)^{q}-q \lambda_{i} s_{i}+c_{q, i} \lambda_{i}^{q} l_{i}^{q}}}{\alpha_{i}-\beta_{i}}\left\|u_{1}-u_{2}\right\|+\frac{\lambda_{i} m_{i}}{\alpha_{i}-\beta_{i}}\left\|v_{1}-v_{2}\right\| .
$$

Put

$$
\theta_{1}^{i}=\frac{\sqrt[q]{\left(r_{i}+t_{i}\right)^{q}-q \lambda_{i} s_{i}+c_{q, i} \lambda_{i}^{q} l_{i}^{q}}}{\alpha_{i}-\beta_{i}}, \quad \theta_{2}^{i}=\frac{\lambda_{i} m_{i}}{\alpha_{i}-\beta_{i}}
$$

Define $\|\cdot\|$ on $\underbrace{X \times \cdots \times X}_{N \text {-times }}$ by $\left\|\left(x_{1}, \ldots, x_{N}\right)\right\|=\left\|x_{1}\right\|+\cdots\left\|x_{N}\right\|$ for all $\left(x_{1}, \ldots, x_{N}\right) \in \underbrace{X \times \cdots \times X}_{N \text {-times }}$. It is easy to see that $(\underbrace{X \times \cdots \times X}_{N \text {-times }},\|\cdot\|)$ is a Banach space. For any given $x_{1}, \ldots, x_{N} \in X$, we choose a finite sequence $w_{1} \in U_{1}\left(x_{N}\right), \ldots, w_{N} \in U_{N}\left(x_{1}\right)$. Define $Q: \underbrace{X \times \cdots \times X}_{N \text {-times }} \rightarrow \underbrace{X \times \cdots \times X}_{N \text {-times }}$ by $Q\left(x_{1}, \ldots, x_{N}\right)=\left(F_{1}\left(x_{1}, w_{1}\right), \ldots, F_{N}\left(x_{N}, w_{N}\right)\right)$. Set $k=\max \left\{\left(\theta_{1}^{1}+\theta_{2}^{N} L_{N}\right), \ldots,\left(\theta_{2}^{1} L_{1}+\right.\right.$ $\left.\left.\theta_{1}^{N}\right)\right\}$, where $L_{1}, \ldots, L_{N}$ are contraction constants of $U_{1}, \ldots, U_{N}$, respectively. We note that $\theta_{1}^{i}+\theta_{2}^{i} L_{i}<\theta_{1}^{i}+\theta_{2}^{i}<1$, for all $i=1,2, \ldots, N$, and so $k<1$. Let $x_{1}, \ldots, x_{N} \in X$, 
$w_{1} \in U_{1}\left(x_{N}\right), \ldots, w_{N} \in U_{N}\left(x_{1}\right)$ and $y_{1}, \ldots, y_{N} \in X, z_{1} \in U_{1}\left(y_{N}\right), \ldots, z_{N} \in U_{N}\left(y_{1}\right)$. By (A3), we get

$$
\begin{aligned}
\left\|Q\left(x_{1}, \ldots, x_{N}\right)-Q\left(y_{1}, \ldots, y_{N}\right)\right\|= & \|\left(F_{1}\left(x_{1}, w_{1}\right), \ldots, F_{N}\left(x_{N}, w_{N}\right)\right) \\
& -\left(F_{1}\left(y_{1}, z_{1}\right), \ldots, F_{N}\left(y_{N}, z_{N}\right)\right) \| \\
= & \left\|F_{1}\left(x_{1}, w_{1}\right)-F_{1}\left(y_{1}, z_{1}\right)\right\| \\
& +\cdots+\left\|F_{N}\left(x_{N}, w_{N}\right)-F_{N}\left(y_{N}, z_{N}\right)\right\| \\
\leq & \left(\theta_{1}^{1}\left\|x_{1}-y_{1}\right\|+\theta_{2}^{1}\left\|w_{1}-z_{1}\right\|\right) \\
& +\cdots+\left(\theta_{1}^{N}\left\|x_{N}-y_{N}\right\|+\theta_{2}^{N}\left\|w_{N}-z_{N}\right\|\right) \\
\leq & \left(\theta_{1}^{1}\left\|x_{1}-y_{1}\right\|+\theta_{2}^{1} L_{1}\left\|x_{N}-y_{N}\right\|\right) \\
& +\cdots+\left(\theta_{1}^{N}\left\|x_{N}-y_{N}\right\|+\theta_{2}^{N} L_{N}\left\|x_{1}-y_{1}\right\|\right) \\
= & \left(\theta_{1}^{1}+\theta_{2}^{N} L_{N}\right)\left\|x_{1}-y_{1}\right\| \\
& +\cdots+\left(\theta_{1}^{N}+\theta_{2}^{1} L_{1}\right)\left\|x_{N}-y_{N}\right\| \\
\leq & k\left\|x_{1}-y_{1}\right\|+\cdots+k\left\|x_{N}-y_{N}\right\| \\
= & k\left(\left\|x_{1}-y_{1}\right\|+\cdots+\left\|x_{N}-y_{N}\right\|\right) \\
= & k\left\|\left(x_{1}, \ldots, x_{N}\right)-\left(y_{1}, \ldots, y_{N}\right)\right\|,
\end{aligned}
$$

and so $Q$ is a contraction on $\underbrace{X \times \cdots \times X}_{N \text {-times }}$. Hence there exists $a_{1}, \ldots, a_{N} \in X, u_{1} \in U_{1}$ $\left(a_{N}\right), \ldots, u_{N} \in U_{N}\left(a_{1}\right)$ such that $a_{1}=F_{1}\left(a_{1}, u_{1}\right), \ldots, a_{N}=F_{N}\left(a_{N}, u_{N}\right)$. From Theorem 3.1, $a_{1}, \ldots, a_{N} \in X, u_{1} \in U_{1}\left(a_{N}\right), \ldots, u_{N} \in U_{N}\left(a_{1}\right)$ is the solution of the problem (3.1).

Theorem 3.4. Let $X$ be q-uniformly smooth real Banach space. For $i=1,2, \ldots, N$. Let $A_{i}, B_{i}$ : $X \rightarrow X$ be single-valued operators, $H_{i}: X \times X \rightarrow X$ single-valued operator satisfy (A1) and suppose that $M_{i}, U_{i}, H_{i}\left(A_{i}, B_{i}\right), S_{i}, S_{i}(\cdot, u)$ satisfy conditions (A2)-(A6), respectively. Then, for any $i \in\{1,2, \ldots, N\}$, the sequences $\left\{a_{n}^{i}\right\}_{n=1}^{\infty}$ and $\left\{u_{n}^{i}\right\}_{n=1}^{\infty}$ generated by Algorithm 3.2 converge strongly to $a_{i}, u_{i} \in U_{i}\left(a_{N-(i-1)}\right)$, respectively.

Proof. By Theorem 3.3, the problem (3.1) has a solution $a_{1}, \ldots, a_{N} \in X, u_{1} \in U_{1}\left(a_{N}\right), \ldots, u_{N} \in$ $U_{N}\left(a_{1}\right)$. From Theorem 3.1, we note that

$$
a_{i}=\sigma_{n} a_{i}+\left(1-\sigma_{n}\right) R_{M_{i}, \lambda_{i}}^{H_{i}(\cdot)}\left[H_{i}\left(A_{i}\left(a_{i}\right), B_{i}\left(a_{i}\right)-\lambda_{i} S_{i}\left(a_{i}, u_{i}\right)\right)\right]
$$


Journal of Applied Mathematics

11

for all $i=1,2, \ldots, N$. Hence, by (3.8) and (3.22), we have

$$
\begin{aligned}
& \left\|a_{n+1}^{i}-a_{n}^{i}\right\|=\| \sigma_{n} a_{n}^{i}+\left(1-\sigma_{n}\right) R_{M_{i}, \lambda_{i}}^{H_{i}(\cdot, \cdot)}\left[H_{i}\left(A_{i}\left(a_{n}^{i}\right), B_{i}\left(a_{n}^{i}\right)\right)-\lambda_{i} S_{i}\left(a_{n}^{i}, u_{n}^{i}\right)\right] \\
& -\left[\sigma_{n} a_{n-1}^{i}+\left(1-\sigma_{n}\right) R_{M_{i}, \lambda_{i}}^{H_{i}(\cdot)}\left[H_{i}\left(A_{i}\left(a_{n-1}^{i}\right), B_{i}\left(a_{n-1}^{i}\right)\right)-\lambda_{i} S_{i}\left(a_{n-1}^{i}, u_{n-1}^{i}\right)\right]\right] \| \\
& \leq \sigma_{n}\left\|a_{n}^{i}-a_{n-1}^{i}\right\|+\left(1-\sigma_{n}\right) \| R_{M_{i}, \lambda_{i}}^{H_{i}(, \cdot)}\left[H_{i}\left(A_{i}\left(a_{n}^{i}\right), B_{i}\left(a_{n}^{i}\right)\right)-\lambda_{i} S_{i}\left(a_{n}^{i}, u_{n}^{i}\right)\right] \\
& -R_{M_{i}, \lambda_{i}}^{H_{i}(\cdot)}\left[H_{i}\left(A_{i}\left(a_{n-1}^{i}\right), B_{i}\left(a_{n-1}^{i}\right)\right)\right. \\
& \left.-\lambda_{i} S_{i}\left(a_{n-1}^{i}, u_{n-1}^{i}\right)\right] \| \\
& =\sigma_{n}\left\|a_{n}^{i}-a_{n-1}^{i}\right\|+\left(1-\sigma_{n}\right) \| R_{M_{i}, \lambda_{i}}^{H_{i}(\cdot, \cdot)}\left[J_{i}\left(a_{n}^{i}, a_{n}^{i}\right)-\lambda_{i} S_{i}\left(a_{n}^{i}, u_{n}^{i}\right)\right] \\
& -R_{M_{i}, \lambda_{i}}^{H_{i}(\cdot, \cdot)}\left[J_{i}\left(a_{n-1}^{i}, a_{n-1}^{i}\right)-\lambda_{i} S_{i}\left(a_{n-1}^{i}, u_{n-1}^{i}\right)\right] \| \\
& \leq \sigma_{n}\left\|a_{n}^{i}-a_{n-1}^{i}\right\|+\left(1-\sigma_{n}\right) \frac{1}{\alpha_{i}-\beta_{i}} \|\left[J_{i}\left(a_{n}^{i}, a_{n}^{i}\right)-\lambda_{i} S_{i}\left(a_{n}^{i}, u_{n}^{i}\right)\right] \\
& -\left[J_{i}\left(a_{n-1}^{i}, a_{n-1}^{i}\right)-\lambda_{i} S_{i}\left(a_{n-1}^{i}, u_{n-1}^{i}\right)\right] \| \\
& =\sigma_{n}\left\|a_{n}^{i}-a_{n-1}^{i}\right\|+\left(1-\sigma_{n}\right) \frac{1}{\alpha_{i}-\beta_{i}} \|\left[J_{i}\left(a_{n}^{i}, a_{n}^{i}\right)-J_{i}\left(a_{n-1}^{i}, a_{n-1}^{i}\right)\right] \\
& -\lambda_{i}\left[S_{i}\left(a_{n}^{i}, u_{n}^{i}\right)-S_{i}\left(a_{n-1}^{i}, u_{n-1}^{i}\right)\right] \| \\
& \leq \sigma_{n}\left\|a_{n}^{i}-a_{n-1}^{i}\right\|+\left(1-\sigma_{n}\right) \frac{1}{\alpha_{i}-\beta_{i}} \|\left[J_{i}\left(a_{n}^{i}, a_{n}^{i}\right)-J_{i}\left(a_{n-1}^{i}, a_{n-1}^{i}\right)\right] \\
& -\lambda_{i}\left[S_{i}\left(a_{n}^{i}, u_{n}^{i}\right)-S_{i}\left(a_{n-1}^{i}, u_{n}^{i}\right)\right] \| \\
& +\left(1-\sigma_{n}\right) \frac{1}{\alpha_{i}-\beta_{i}}\left\|S_{i}\left(a_{n-1}^{i}, u_{n}^{i}\right)-S_{i}\left(a_{n-1}^{i}, u_{n-1}^{i}\right)\right\| .
\end{aligned}
$$

By Lemma 2.4, we obtain

$$
\begin{aligned}
&\left\|J_{i}\left(a_{n}^{i}, a_{n}^{i}\right)-J_{i}\left(a_{n-1}^{i}, a_{n-1}^{i}\right)-\lambda_{i}\left[S_{i}\left(a_{n}^{i}, u_{n}^{i}\right)-S_{i}\left(a_{n-1}^{i}, u_{n}^{i}\right)\right]\right\|^{q} \\
& \leq\left\|J_{i}\left(a_{n}^{i}, a_{n}^{i}\right)-J_{i}\left(a_{n-1}^{i}, a_{n-1}^{i}\right)\right\|^{q} \\
& \quad-q \lambda_{i}\left\langle S_{i}\left(a_{n}^{i}, u_{n}^{i}\right)-S_{i}\left(a_{n-1}^{i}, u_{n}^{i}\right), J_{q, i}\left(J_{i}\left(a_{n}^{i}, a_{n}^{i}\right)-J_{i}\left(a_{n-1}^{i}, a_{n-1}^{i}\right)\right)\right\rangle \\
&+c_{q, i} \lambda_{i}^{q}\left\|S_{i}\left(a_{n}^{i}, u_{n}^{i}\right)-S_{i}\left(a_{n-1}^{i}, u_{n}^{i}\right)\right\|^{q} .
\end{aligned}
$$


12

Journal of Applied Mathematics

From (A4), we note that

$$
\begin{aligned}
\left\|J_{i}\left(a_{n}^{i}, a_{n}^{i}\right)-J_{i}\left(a_{n-1}^{i}, a_{n-1}^{i}\right)\right\|= & \left\|H_{i}\left(A_{i}\left(a_{n}^{i}\right), B_{i}\left(a_{n}^{i}\right)\right)-H_{i}\left(A_{i}\left(a_{n-1}^{i}\right), B_{i}\left(a_{n-1}^{i}\right)\right)\right\| \\
\leq & \left\|H_{i}\left(A_{i}\left(a_{n}^{i}\right), B_{i}\left(a_{n}^{i}\right)\right)-H_{i}\left(A_{i}\left(a_{n-1}^{i}\right), B_{i}\left(a_{n}^{i}\right)\right)\right\| \\
& +\left\|H_{i}\left(A_{i}\left(a_{n-1}^{i}\right), B_{i}\left(a_{n}^{i}\right)\right)-H_{i}\left(A_{i}\left(a_{n-1}^{i}\right), B_{i}\left(a_{n-1}^{i}\right)\right)\right\| \\
\leq & \left(r_{\mathrm{i}}+t_{i}\right)\left\|a_{n}^{i}-a_{n-1}^{i}\right\| .
\end{aligned}
$$

From (3.24) and (A6), it follows that

$$
-q \lambda_{i}\left\langle S_{i}\left(a_{n}^{i}, u_{n}^{i}\right)-S_{i}\left(a_{n-1}^{i}, u_{n}^{i}\right), J_{q, 1}\left(J_{i}\left(a_{n}^{i}, a_{n}^{i}\right)-J_{i}\left(a_{n-1}^{i}, a_{n-1}^{i}\right)\right)\right\rangle \leq-q \lambda_{i} S_{i}\left\|a_{n}^{i}-a_{n-1}^{i}\right\|^{q} .
$$

By (3.23), (3.24), and (A5), we have

$$
\begin{aligned}
\left\|S_{i}\left(a_{n-1}^{i}, u_{n}^{i}\right)-S_{i}\left(a_{n-1}^{i}, u_{n-1}^{i}\right)\right\| & \leq m_{i}\left\|u_{n}^{i}-u_{n-1}^{i}\right\| \\
& \leq m_{i} d_{i}\left(1+n^{-1}\right)\left\|a_{n}^{i}-a_{n-1}^{i}\right\|, \\
\left\|S_{i}\left(a_{n}^{i}, u_{n}^{i}\right)-S_{i}\left(a_{n-1}^{i}, u_{n}^{i}\right)\right\| & \leq l_{i}\left\|a_{n}^{i}-a_{n-1}^{i}\right\| .
\end{aligned}
$$

From (3.23)-(3.28), we obtain

$$
\begin{gathered}
\left\|J_{i}\left(a_{n}^{\mathrm{i}}, a_{n}^{i}\right)-J_{i}\left(a_{n-1}^{i}, a_{n-1}^{i}\right)-\lambda_{i}\left[S_{i}\left(a_{n}^{i}, u_{n}^{i}\right)-S_{i}\left(a_{n-1}^{i}, u_{n}^{i}\right)\right]\right\|^{q} \\
\leq \frac{\sqrt[q]{\left(r_{i}+t_{i}\right)^{q}-q \lambda_{i} s_{i}+c_{q, i} \lambda_{i}^{q} l_{i}^{q}}}{\alpha_{i}-\beta_{i}}\left\|a_{n}^{i}-a_{n-1}^{i}\right\| \\
\quad+\frac{\lambda_{i} m_{i}}{\alpha_{i}-\beta_{i}} d_{i}\left(1+n^{-1}\right)\left\|a_{n}^{i}-a_{n-1}^{i}\right\| .
\end{gathered}
$$

Hence, by (3.23), (3.28) and (3.29), we have

$$
\begin{aligned}
\left\|a_{n+1}^{i}-a_{n}^{i}\right\| \leq & \sigma_{n}\left\|a_{n}^{i}-a_{n-1}^{i}\right\|+\left(1-\sigma_{n}\right) \frac{\sqrt[q]{\left(r_{i}+t_{i}\right)^{q}-q \lambda_{i} s_{i}+c_{q, i} \lambda_{i}^{q} l_{i}^{q}}}{\alpha_{i}-\beta_{i}}\left\|a_{n}^{i}-a_{n-1}^{i}\right\| \\
& +\left(1-\sigma_{n}\right) \frac{\lambda_{i} m_{i}}{\alpha_{i}-\beta_{i}} d_{i}\left(1+n^{-1}\right)\left\|a_{n}^{i}-a_{n-1}^{i}\right\| .
\end{aligned}
$$

Put $k=\max \left\{\pi_{1} \ldots, \pi_{N}\right\}$, where

$$
\pi_{i}=\frac{\sqrt[q]{\left(r_{i}+t_{i}\right)^{q}-q \lambda_{i} s_{i}+c_{q, i} \lambda_{i}^{q} l_{i}^{q}}}{\alpha_{i}-\beta_{i}}+\frac{\lambda_{i} m_{i} d_{i}\left(1+n^{-1}\right)}{\alpha_{i}-\beta_{i}} .
$$


It follows from (3.30) that

$$
\begin{aligned}
\left\|a_{n+1}^{1}-a_{n}^{1}\right\|+\cdots+\left\|a_{n+1}^{N}-a_{n}^{N}\right\| \leq & \sigma_{n}\left\|a_{n}^{1}-a_{n-1}^{1}\right\|+\left(1-\sigma_{n}\right) k\left\|a_{n}^{1}-a_{n-1}^{1}\right\| \\
& +\cdots+\sigma_{n}\left\|a_{n}^{N}-a_{n-1}^{N}\right\|+\left(1-\sigma_{n}\right) k\left\|a_{n}^{N}-a_{n-1}^{N}\right\| .
\end{aligned}
$$

Set $c_{n}=\left\|a_{n}^{1}-a_{n-1}^{1}\right\|+\cdots+\left\|a_{n}^{N}-a_{n-1}^{N}\right\|$ and $k_{n}=k+(1-k) \sigma_{n}$. From (3.32), we obtain

$$
c_{n+1} \leq k_{n} c_{n}, \quad \forall n=0,1,2, \ldots
$$

Since $\lim \sup _{n \rightarrow \infty} \sigma_{n}<1$, we have $\limsup _{n \rightarrow \infty} k_{n}<1$. Thus, it follows from Lemma 2.8 that $c_{n+1} \rightarrow 0$ and hence $\lim _{n \rightarrow \infty}\left\|a_{n+1}^{i}-a_{n}^{i}\right\|=0$. Therefore, $\left\{a_{n}^{i}\right\}$ is a Cauchy sequence and hence there exists $a_{i} \in X$ such that $a_{n}^{i} \rightarrow a_{i}$ as $n \rightarrow \infty$, for all $i=1,2, \ldots, N$. Next, we will show that $u_{n}^{1} \rightarrow u_{1} \in U_{1}\left(a_{N}\right)$ as $n \rightarrow \infty$. Hence, it follows from (3.9) that $\left\{u_{n}^{1}\right\}$ is also a Cauchy sequence. Thus there exists $u_{1} \in X$ such that $u_{n}^{1} \rightarrow u_{1}$ as $n \rightarrow \infty$. Consider

$$
\begin{aligned}
d\left(u_{1}, U_{1}\left(a_{N}\right)\right) & =\inf \left\{\left\|u_{1}-q\right\|: q \in U_{1}\left(a_{N}\right)\right\} \\
& \leq\left\|u_{1}-u_{n}^{1}\right\|+d\left(u_{n}^{1}, U_{1}\left(a_{N}\right)\right) \\
& \leq\left\|u_{1}-u_{n}^{1}\right\|+D\left(U_{1}\left(a_{n}^{N}\right), U_{1}\left(a_{N}\right)\right) \\
& \leq\left\|u_{1}-u_{n}^{1}\right\|+d_{1}\left\|a_{n}^{N}-a_{N}\right\| \longrightarrow 0
\end{aligned}
$$

as $n \rightarrow \infty$. Since $U_{1}\left(a_{N}\right)$ is a closed set and $d\left(u_{1}, U_{1}\left(a_{N}\right)\right)=0$, we have $u_{1} \in U_{1}\left(a_{N}\right)$. By continuing the above process, there exist $u_{2} \in U_{2}\left(a_{N-1}\right), \ldots, u_{N} \in U_{N}\left(a_{1}\right)$ such that $u_{n}^{2} \rightarrow$ $u_{2}, \ldots, u_{n}^{N} \rightarrow u_{N}$ as $n \rightarrow \infty$. Hence, by (3.8), we obtain

$$
a_{i}=R_{M_{i}, \lambda_{i}}^{H_{i}(\cdot)}\left[H_{i}\left(A_{i}\left(a_{i}\right), B_{i}\left(a_{i}\right)\right)-\lambda_{i} S_{i}\left(a_{i}, u_{i}\right)\right]
$$

Therefore, it follows from Theorem 3.1 that $a_{1}, \ldots, a_{N}$ is a solution of problem (3.1).

Setting $N=2$ in Theorem 3.3, we have the following result.

Corollary 3.5. Let $X$ be q-uniformly smooth real Banach spaces. Let $A_{i}, B_{i}: X \rightarrow X$ be singled valued operators, $H_{i}: X \times X \rightarrow X$ a single-valued operator such that $H\left(A_{i}, B_{i}\right)$ is $\alpha_{i}$-strongly accretive with respect to $A_{i}, \beta_{i}$-relaxed accretive with respect to $B_{i}$ and $\alpha_{i}>\beta_{i}$ and suppose that $M_{i}$ : $X \rightarrow 2^{X}$ is an $H_{i}(\cdot, \cdot)$-accretive set-valued mapping and $U_{i}: X \rightarrow C(X)$ contraction set-valued mapping with $0 \leq L_{i}<1$ and nonempty values, for all $i=1,2$. Assume that $H_{i}\left(A_{i}, B_{i}\right)$ is $r_{i}$-Lipschitz continuous with respect to $A_{i}$ and $t_{i}$-Lipschitz continuous with respect to $B_{i}, S_{i}: X \times X \rightarrow X$ is $l_{i}$-Lipschitz continuous with respect to its first argument and $m_{i}$-Lipschitz continuous with respect 
to its second argument, $S_{1}(\cdot, y)$ is $S_{1}$-strongly accretive with respect to $H_{1}\left(A_{1}, B_{1}\right)$, and $S_{2}(x, \cdot)$ is $s_{2}$-strongly accretive with respect to $\mathrm{H}_{2}\left(A_{2}, B_{2}\right)$, for all $i=1,2$. If

$$
\frac{\sqrt[q]{\left(r_{i}+t_{i}\right)^{q}-q \lambda_{i} s_{i}+c_{q, i} i \lambda_{i}^{q} l_{i}^{q}}}{\alpha_{i}-\beta_{i}}+\frac{\lambda_{i} m_{i}}{\alpha_{i}-\beta_{i}}<1,
$$

for all $i \in\{1,2\}$, then problem (3.2) has a solution $a_{1}, a_{2} \in X, u_{1} \in U_{1}\left(a_{2}\right), u_{2} \in U_{2}\left(a_{1}\right)$.

Setting $N=1$ in Theorem 3.3, we have the following result.

Corollary 3.6. Let $X$ be q-uniformly smooth real Banach spaces. Let $A, B: X \rightarrow X$ be two singled valued operators, $H: X \times X \rightarrow X$ a single-valued operator such that $H(A, B)$ is $\alpha$-strongly accretive with respect to $A$, $\beta$-relaxed accretive with respect to $B$, and $\alpha>\beta$ and suppose that $M: X \rightarrow 2^{X}$ is an $H(\cdot, \cdot)$-accretive set-valued mapping, $U: X \rightarrow C(X)$ is contraction set-valued mapping with $0 \leq L<1$ and nonempty values. Assume that $H(A, B)$ is $r$-Lipschitz continuous with respect to $A$ and $t$-Lipschitz continuous with respect to $B, S: X \times X \rightarrow X$ is l-Lipschitz continuous with respect to its first argument and $m$-Lipschitz continuous with respect to its second argument, $S(\cdot, y)$ is s-strongly accretive with respect to $H(A, B)$. If

$$
\frac{\sqrt[q]{(r+t)^{q}-q \lambda s+c_{q}, \lambda q l^{q}}}{\alpha-\beta}+\frac{\lambda m}{\alpha-\beta}<1,
$$

then problem (3.3) has a solution $a \in X$ and $u \in U(a)$.

\section{Acknowledgments}

The first author would like to thank the Office of the Higher Education Commission, Thailand, financial support under Grant CHE-Ph.D-THA-SUP/191/2551, Thailand. Moreover, the second author would like to thank the Thailand Research Fund for financial support under Grant BRG5280016.

\section{References}

[1] A. Hassouni and A. Moudafi, "A perturbed algorithm for variational inclusions," Journal of Mathematical Analysis and Applications, vol. 185, no. 3, pp. 706-712, 1994.

[2] S. Adly, "Perturbed algorithms and sensitivity analysis for a general class of variational inclusions," Journal of Mathematical Analysis and Applications, vol. 201, no. 2, pp. 609-630, 1996.

[3] X. P. Ding, "Perturbed proximal point algorithms for generalized quasivariational inclusions," Journal of Mathematical Analysis and Applications, vol. 210, no. 1, pp. 88-101, 1997.

[4] K. R. Kazmi, "Mann and Ishikawa type perturbed iterative algorithms for generalized quasivariational inclusions," Journal of Mathematical Analysis and Applications, vol. 209, no. 2, pp. 572-584, 1997.

[5] Q. H. Ansari and J.-C. Yao, "A fixed point theorem and its applications to a system of variational inequalities," Bulletin of the Australian Mathematical Society, vol. 59, no. 3, pp. 433-442, 1999.

[6] Q. H. Ansari, S. Schaible, and J. C. Yao, "System of vector equilibrium problems and its applications," Journal of Optimization Theory and Applications, vol. 107, no. 3, pp. 547-557, 2000.

[7] S. Plubtieng and K. Sombut, "Weak convergence theorems for a system of mixed equilibrium problems and nonspreading mappings in a Hilbert space," Journal of Inequalities and Applications, vol. 2010, Article ID 246237, 12 pages, 2010. 
[8] S. Plubtieng and K. Sitthithakerngkiet, "On the existence result for system of generalized strong vector quasiequilibrium problems," Fixed Point Theory and Applications, vol. 2011, Article ID 475121, 9 pages, 2011.

[9] S. Plubtieng and T. Thammathiwat, "Existence of solutions of systems of generalized implicit vector quasi-equilibrium problems in G-convex spaces," Computers \& Mathematics with Applications, vol. 62, no. 1, pp. 124-130, 2011.

[10] R. U. Verma, "A-monotonicity and applications to nonlinear variational inclusion problems," Journal of Applied Mathematics and Stochastic Analysis, no. 2, pp. 193-195, 2004.

[11] W.-Y. Yan, Y.-P. Fang, and N.-J. Huang, "A new system of set-valued variational inclusions with $H$ monotone operators," Mathematical Inequalities $\mathcal{E}$ Applications, vol. 8, no. 3, pp. 537-546, 2005.

[12] S. Plubtieng and W. Sriprad, "A viscosity approximation method for finding common solutions of variational inclusions, equilibrium problems, and fixed point problems in Hilbert spaces," Fixed Point Theory and Applications, vol. 2009, Article ID 567147, 20 pages, 2009.

[13] R. U. Verma, "General nonlinear variational inclusion problems involving $A$-monotone mappings," Applied Mathematics Letters, vol. 19, no. 9, pp. 960-963, 2006.

[14] Y. J. Cho, H.-Y. Lan, and R. U. Verma, "Nonlinear relaxed cocoercive variational inclusions involving $(A, \eta)$-accretive mappings in Banach spaces," Computers $\mathcal{E}$ Mathematics with Applications, vol. 51, no. 9-10, pp. 1529-1538, 2006.

[15] R. U. Verma, "Approximation solvability of a class of nonlinear set-valued variational inclusions involving $(A, \eta)$-monotone mappings," Journal of Mathematical Analysis and Applications, vol. 337, no. 2, pp. 969-975, 2008.

[16] Y.-Z. Zou and N.-J. Huang, "H(.,.)-accretive operator with an application for solving variational inclusions in Banach spaces," Applied Mathematics and Computation, vol. 204, no. 2, pp. 809-816, 2008.

[17] Y. Z. Zou and N. J. Huang, "A new system of variational inclusions involving $H(\cdot, \cdot)$-accretive operator in Banach spaces," Applied Mathematics and Computation, vol. 212, pp. 135-144, 2009.

[18] H. K. Xu, "Inequalities in Banach spaces with applications," Nonlinear Analysis. Theory, Methods $\mathcal{E}$ Applications, vol. 16, no. 12, pp. 1127-1138, 1991.

[19] Y.-P. Fang, N.-J. Huang, and H. B. Thompson, "A new system of variational inclusions with $(H, \eta)$ monotone operators in Hilbert spaces," Computers \& Mathematics with Applications, vol. 49, no. 2-3, pp. 365-374, 2005.

[20] S. B. Nadler,, "Multi-valued contraction mappings," Pacific Journal of Mathematics, vol. 30, pp. 475-488, 1969. 


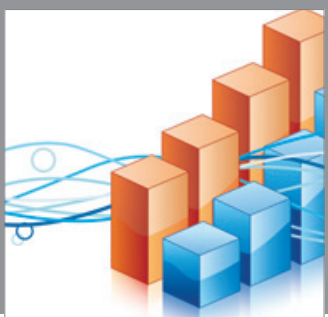

Advances in

Operations Research

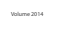

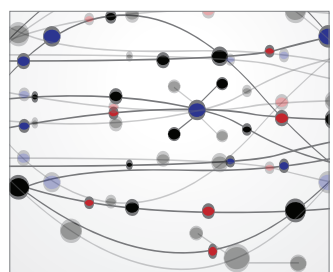

\section{The Scientific} World Journal
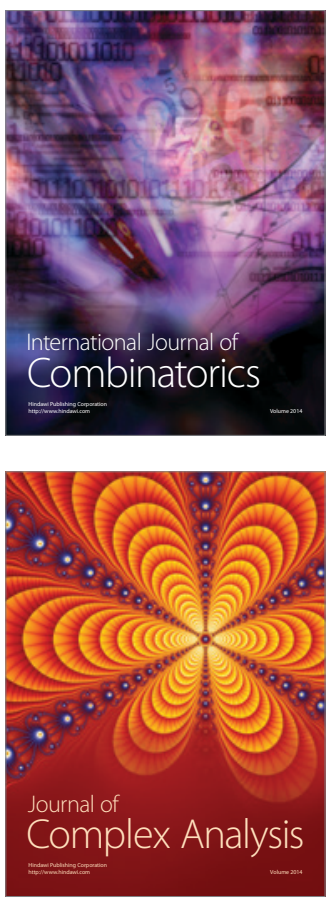

International Journal of

Mathematics and

Mathematical

Sciences
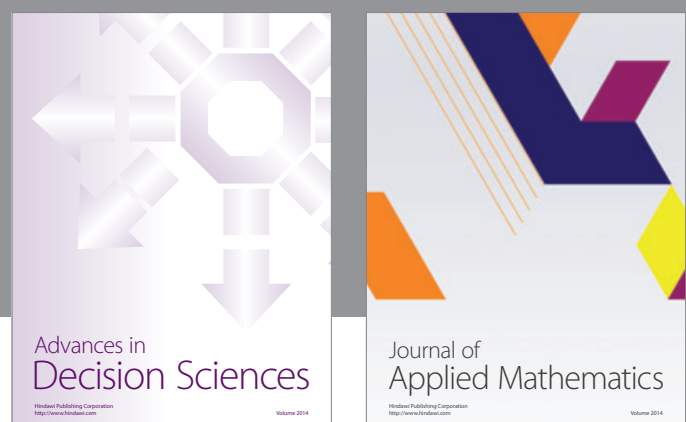

Journal of

Applied Mathematics
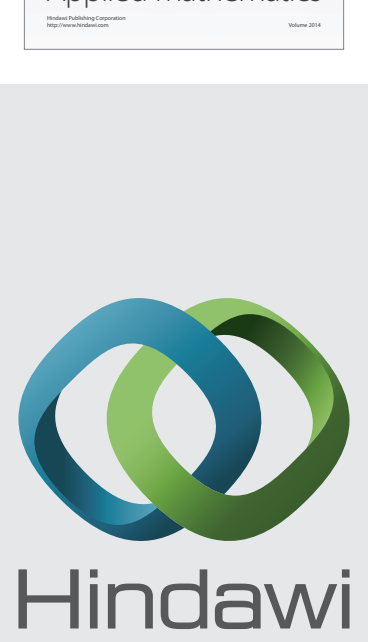

Submit your manuscripts at http://www.hindawi.com
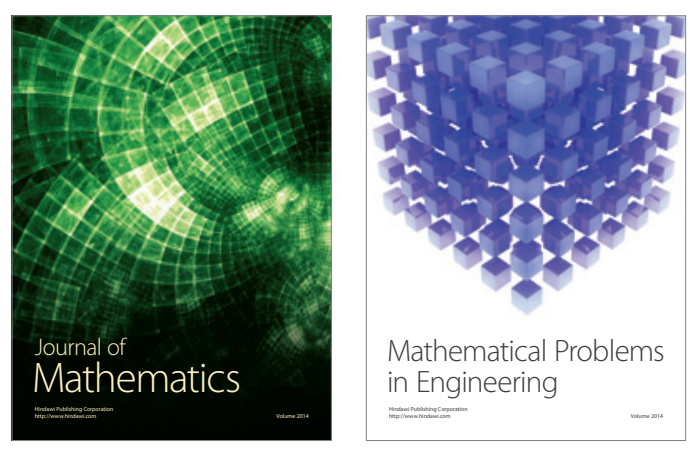

Mathematical Problems in Engineering
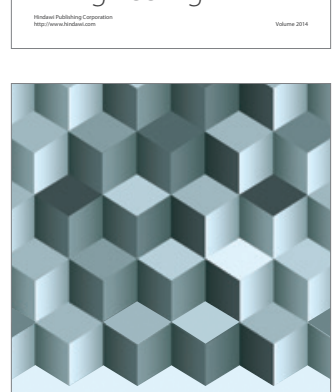

Journal of

Function Spaces
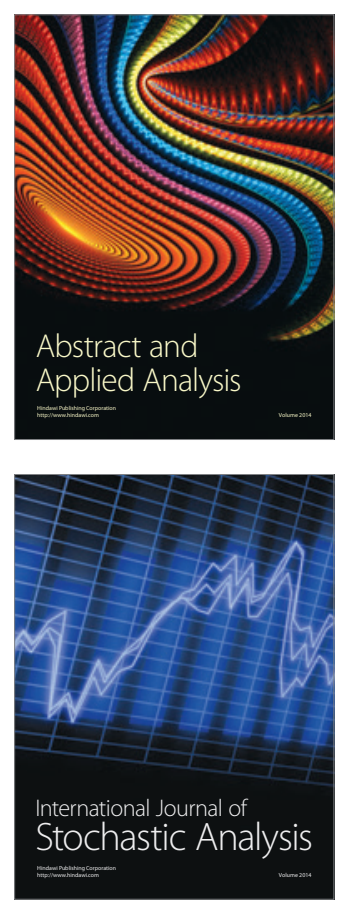

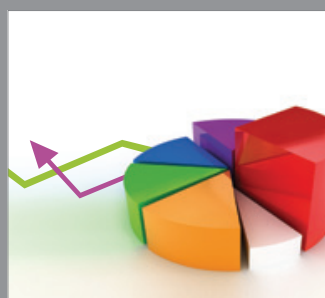

ournal of

Probability and Statistics

Promensencen
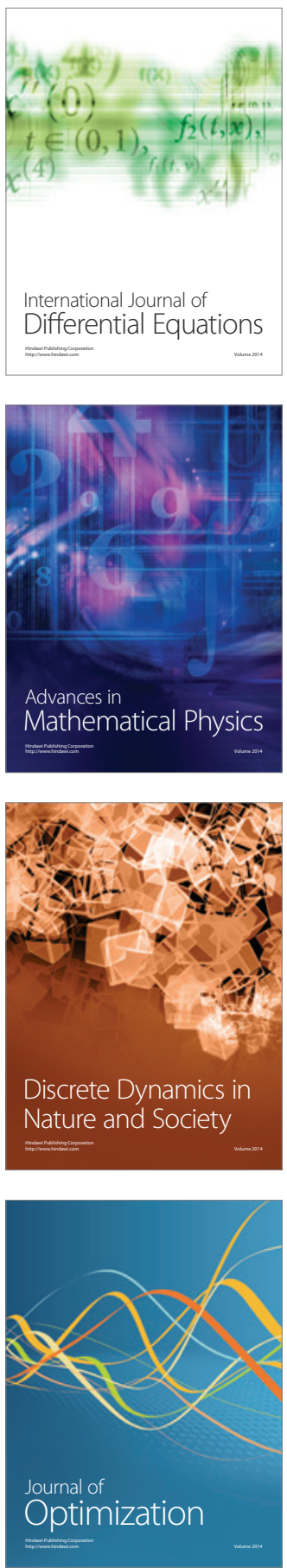